\title{
Triple Negative Breast Cancer Prevalence in Indian Patients over a Decade: A Systematic Review \\ Suvobrata Sarkar ${ }^{1 *}$ and Murtaza Akhtar ${ }^{2}$
}

${ }^{1}$ Assistant Professor, Computer science and engineering, Dr. B.C. Roy Engineering College, Durgapur West Bengal, India ${ }^{2}$ Ex-Professor \& Head, Department of Surgery, N.K.P Salve Institute of Medical Sciences, Nagpur, India

*Corresponding author: Suvobrata Sarkar, Assistant Professor, Computer Science and Engineering, Dr. B.CRoy Engineering College, Durgapur, West Bengal, India

\begin{abstract}
Background: Triple Negative Breast Cancer (TNBC) is an aggressive variant of breast cancer with penurious prognosis and limited therapeutic options. This subtype is characterised with higher incidence rate among the younger women, aggressive tumour behaviour, with early local recurrence and distant metastasis leading to poor outcomes.

Objectives: In India, majority of prevalence data of TNBC is hospital based from patient treated at tertiary care hospitals or centres ranging from $10 \%$ to $43 \%$ which is evident from the various cohort studies reported over a decade. A systematic review and literature oriented meta-analysis is performed to estimate the prevalence of TNBC from the different study specific prevalence.

Methods: The present review was conducted with PubMed and Google search of journal articles published between $1^{\text {st }}$ January 2010 and $31^{\text {st }}$ March 2020 that reported the TNBC prevalence in Indian patients, clinicopathological parameters and their comparison with non-TNBC subtype. Duplication of the data was avoided by excluding meta-analysis and other review articles. Primary studies were chosen based on the inclusion criteria and random effects meta-analysis was performed to obtain the summary estimate from the pooled study-specific estimates.
\end{abstract}

Results: Data for the meta-analysis were obtained from 16 primary studies which involves 9787 Indian patients mostly women treated with the invasive breast cancer. The overall pooled estimate of prevalence in TNBC was $25.04 \%$ (95\% $\mathrm{Cl}, 23.42 \%$ to $26.67 \%$ ). The substantial heterogeneity was observed between the included studies with the values of heterogeneity: $\left(\mathrm{Tau}^{2}=10.99 ; \mathrm{df}=15(P<0.00001) ; I^{2}=\right.$ $100 \%)$. Risk of the publication bias was assessed with the corresponding funnel plots.
Conclusions: According to the pooled estimate prevalence of TNBC in India is $25.04 \%$ which is higher in comparison to the western literature. Hence there is a need for extensive research on TNBC patients to identify the suitable therapeutic regimen for the improved survival.

\section{Keywords}

Heterogeneity, Indian cohorts, Prevalence rate, Random effects meta-analysis, Systematic review, Triple negative breast cancer

\section{Introduction}

The breast cancer [1] is one of the common cancers reported in Indian women and accounts for $14 \%$ of all other type of cancers in India [2,3]. In accordance with the GLOBOCAN data (2018), 1,62,468 new cases were reported with the death occurrence of 87,090 cases [4]. The TNBC is an aggressive variant of breast cancer associated with poor health outcome which is identified by Immuno-Histochemistry (IHC) examination finding of missing expression of Progesterone Receptor (PR), Oestrogen Receptor (ER) and absence of amplification or over expression of the Human Epidermal Growth Factor Receptor 2 (HER 2). It presents with varied clinicopathological and molecular characteristics with poor prognosis and limited therapeutic options. There is a scarcity of the hormone receptor data in the country due to lack of IHC facilities and high cost of IHC estimation as a result of which exact calculation of prevalence of TNBC is not possible. This was the rationale for carrying out present study and this objective was carried out by performing systematic review and meta-analysis.

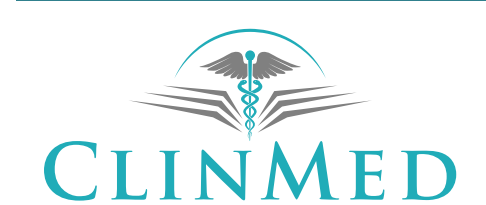

INTERNATIONAL LIBRARY
Citation: Sarkar S, Akhtar M (2022) Triple Negative Breast Cancer Prevalence in Indian Patients over a Decade: A Systematic Review. Int J Clin Biostat Biom 8:045. doi.org/10.23937/2469-5831/1510045 Accepted: January 10, 2022: Published: January 12, 2022

Copyright: (c) 2022 Sarkar S, et al. This is an open-access article distributed under the terms of the Creative Commons Attribution License, which permits unrestricted use, distribution, and reproduction in any medium, provided the original author and source are credited. 


\section{Methods}

\section{Search strategy}

A systematic review was performed with journal articles published between $1^{\text {st }}$ January 2010 and $31^{\text {st }}$ March 2020 available through PubMed and Google that reported the prevalence of the TNBC in the population of India predominantly female patients. The searching and reporting of articles were done through the Preferred Reporting Items for Systematic Reviews and Metaanalyses (PRISMA) specification [5]. The significant search terms include the combination of keywords: Breast cancer, triple negative, TNBC and India. The study eligibility was decided by following inclusion \& exclusion criteria. The inclusion criteria entailed retrospective studies that involve Indian cohorts histo-pathologically diagnosed with breast carcinoma and were published from multiple centres across vast Indian geography.
The studies reporting the triple negative breast cancer prevalence proportion, clinicopathological features associated risk factors and their comparison with the non-TNBC group of patients. The studies with gene expression profiling analysis, bilateral breast cancer and that do not meet the PICOS (participants, interventions, comparisons, outcomes, and study design) criteria [6] were omitted as exclusion criteria. Duplication of data was avoided by excluding meta-analysis and other review articles.

The authors individually screened all the similar titles and abstracts to recognize the potential citation. The full text of all pertinent articles was retrieved and assessed independently by the two authors satisfying the inclusion criteria. In case of disagreements related to a specific article, the matter was left to clinician's judgment. The related journal articles were screened in accordance of predefined inclusion criteria. Searching

\section{PRISMA Flow Diagram}

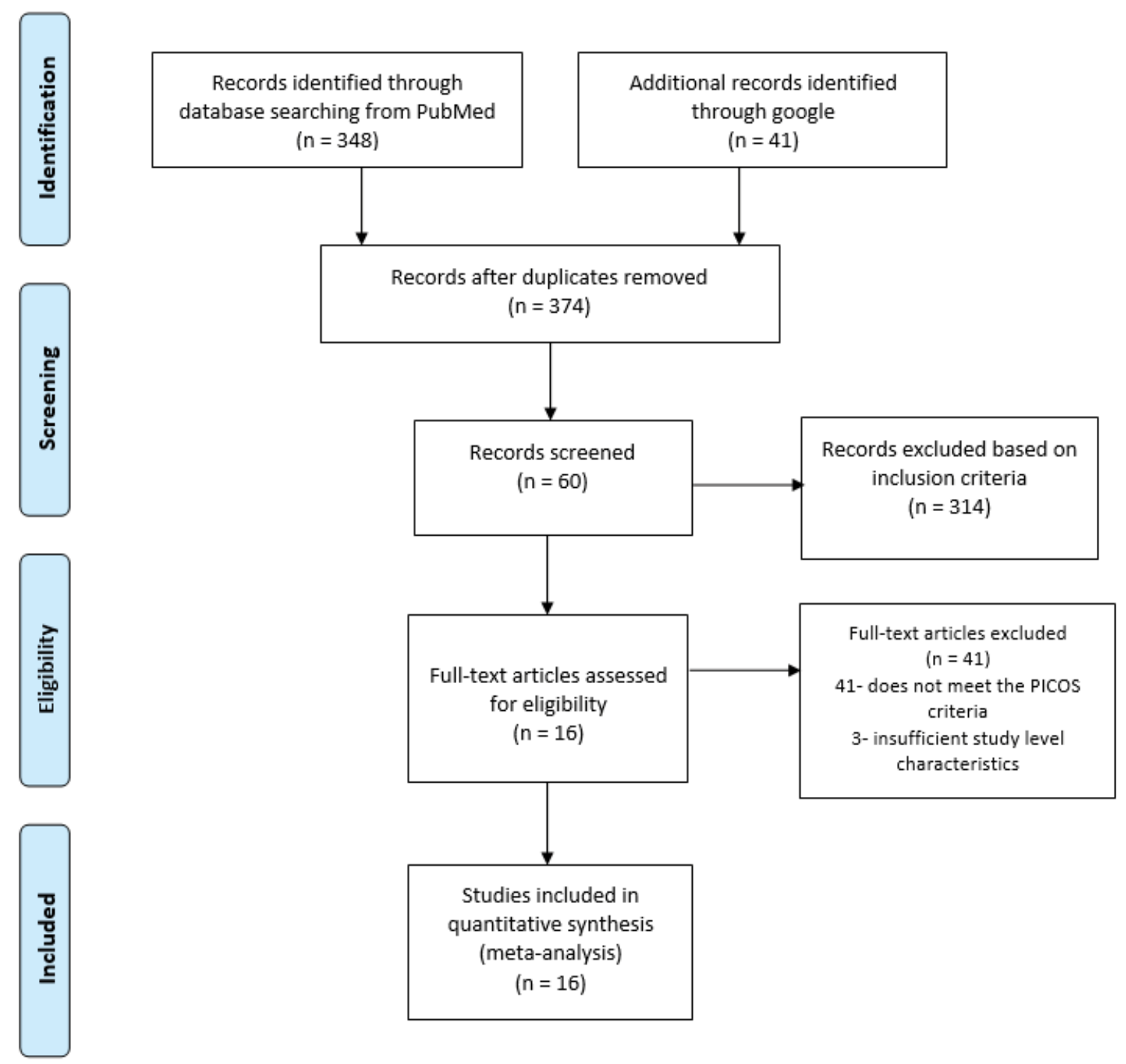

Figure 1: Flowchart to select Indian studies of breast cancer cohorts for estimating the prevalence of TNBC with preferred reporting items for systematic review and meta-analysis (PRISMA). 
and reporting of articles was done based on PRISMA guidelines. The Figure 1 shows the overall flow diagram of study selection. The assessment of study quality was performed as per the of STROBE guidelines [7]. The checklist of items that address the PICOS were investigated in the included studies while reporting the systematic review. The study level characteristics that were considered from each study are: Publication year, number of the TNBC cases, proportion of the TNBC case, mean age of incidence, premenopausal rate, proportion of patients with grade 2 , grade 3 tumours and node positivity involvement. Detail definition of immune histochemistry staining procedure was not discussed in some of the included studies but the patients with negative values of ER, PR and HER2 (IHC score 0 or $1+$ or FISH non-amplified) were considered as triple negative. The patients were evaluated based on these study level characteristics and compared between the TNBC and non-TNBC groups.

\section{Meta-analysis}

The meta-analysis, a statistical method which integrates the study specific estimates from the primary studies and produces the overall summary estimates of an effect was analysed. The random effects metaanalysis model was used to determine the overall effect of prevalence across the 16 included studies and was compared with the fixed effects model. The results were graphically represented on a forest plot which indicates the overall prevalence outcome, studyspecific prevalence rate, standard error of respective studies, weights and the heterogeneity statistics. The heterogeneity index $\left(l^{2}\right)$ statistic [8], which indicates the percentage of variations between the eligible studies, was evaluated to assess the heterogeneity. The $l^{2}$ value greater than $50 \%$ and $P<0.05$ were considered as statistically significant. The source of the heterogeneity was discovered by applying subgroup analyses. The funnel plot had been used for assessing publication bias. It is a scatter plot which estimates the prevalence of TNBC with the corresponding study specific standard error. Rev Man (Review Manager), the Cochrane Collaboration's software is used for performing metaanalysis and representing the results graphically. RevMan can also be utilized for reviewing diagnostic test accuracy studies and study's methodology reviews. In this article, RevMan version 5.3 [9] was used to analyse the performance of meta-analysis.

\section{Results}

A total of 16 Indian studies [10-25] reporting prevalence of TNBC with clinicopathological parameters and treatment outcome were studied and 9787 patients of breast cancer patients were analysed for systematic review. Out of these 16 studies, 7 studies were reported from north India, 7 comes from south India, one each from central India and the east suggesting a pan India representation. The mean age of the patients was 46.2 years SD 3.44 and a range from $38-50$ years. The prevalence of Nottingham Grade 3 disease an indicator of aggressive pathology ranged from $28.10 \%$ to $96 \%$ with a mean of $59.25 \%$ and standard deviation 21 . These finding suggested prevalence of more aggressive disease in Indian females.

The random effects meta-analysis model was evaluated to determine the combined estimate of prevalence across the studies and the inverse-variance estimation method was taken into consideration. Overall pooled prevalence of the TNBC was $25.04 \%$ $(95 \% \mathrm{Cl}, 23.42 \%$ to $26.67 \%)$ with effect measure of

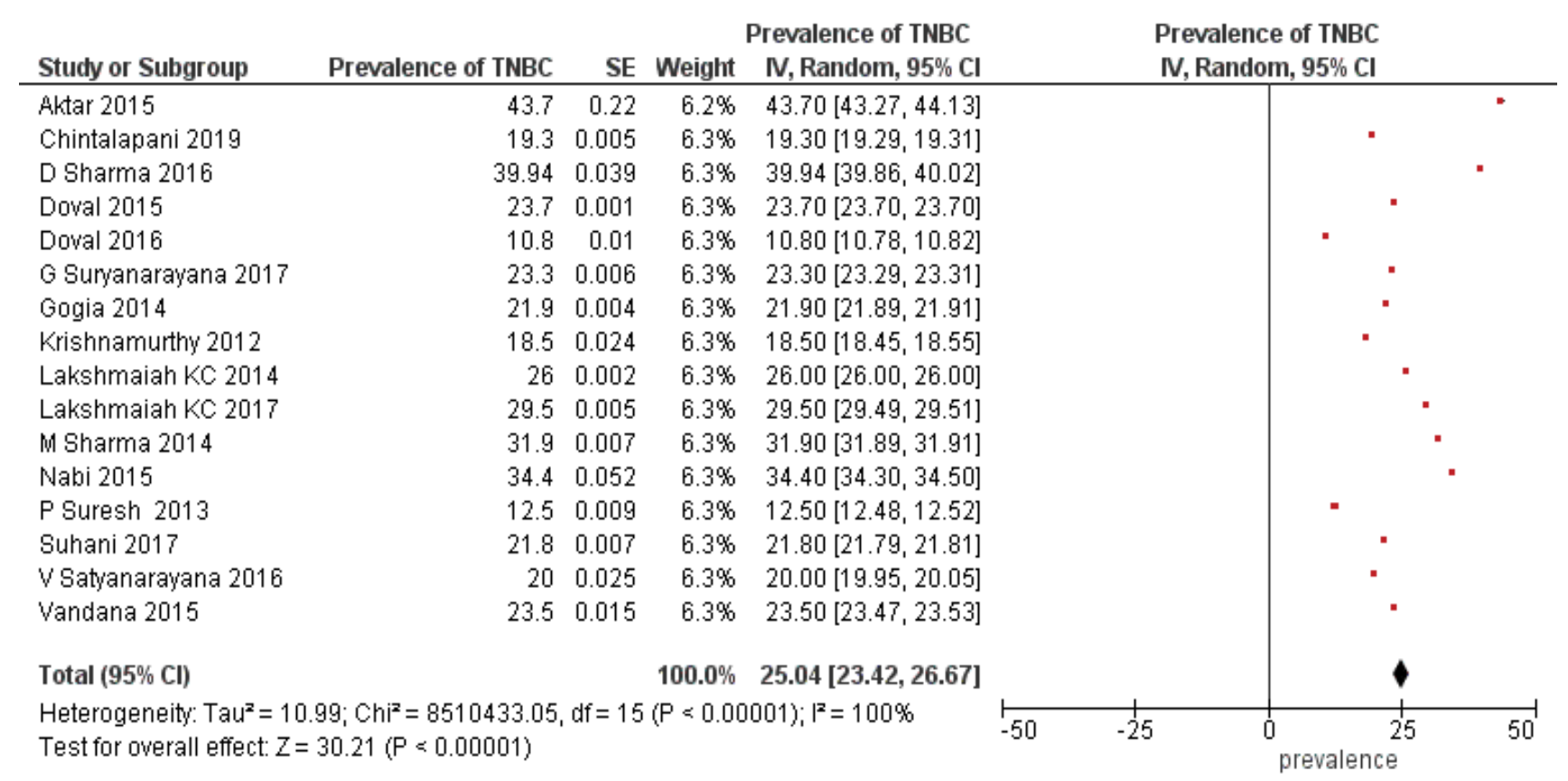

Figure 2: Forest plot-overall prevalence rate of TNBC in Indian cohorts with random effects model. 
TNBC prevalence (Figure 2). For comparison, fixed effects meta-analysis method was analysed with pooled prevalence estimate $23.95 \%$ ( $95 \% \mathrm{Cl}, 23.94 \%$ to $23.95 \%$ ) and the results also showed significant changes in the over effect of the Z-score (Figure 3). The substantial heterogeneity was observed across the studies with the random effects values of heterogeneity $\left(\mathrm{Tau}^{2}=10.99\right.$; $\mathrm{df}$ $\left.=15(P<0.00001) ; I^{2}=100 \%\right)$. In subgroup analysis, the studies were categorized into 4 regions: North, south, central and east. The heterogeneity was not applicable for central and eastern region due to the presence of single study in each location. However, significant heterogeneity was observed between southern and northern region with 7 included studies in each location. The results obtained for subgroup differences: $\mathrm{Chi}^{2}=$ $2942.78, \mathrm{df}=3(P<0.00001), l^{2}=99.9 \%$ as shown in Figure 4.

Depending upon the number of TNBC cases in the included studies, the studies were divided into two subgroups; TNBC cases $<150$ and TNBC cases $>150$ ) to find the overall effect of between-study heterogeneity. There were 10 studies in TNBC cases $<150$ and 6 TNBC studies greater than 150 cases. The overall random effect of TNBC cases < 150 was compared with TNBC cases > 150 with results; TNBC cases $<150$ heterogeneity: Tau $^{2}$ $=24.76 ; \mathrm{df}=9(P<0.00001) ; l^{2}=100 \%$ versus TNBC cases $>150$ heterogeneity: $\mathrm{Tau}^{2}=16.49 ; \mathrm{df}=5(P<0.00001)$; $I^{2}=100 \%$ (shown in Figure 5 ). The corresponding value of test for sub group differences: $\mathrm{Chi}^{2}=1.79, \mathrm{df}=1(P=$ $0.18), I^{2}=44.1 \%$ and was found statistically insignificant.

The funnel plot is commonly known for its ability to discover the publication bias. These plots are useful in visual inspection of data exploration and identifying the absence of asymmetry [26]. Apart from the publication bias, the heterogeneity between the studies can cause asymmetry in the funnel plot. The funnel plot with graphical interpretation in random effects model of TNBC prevalence showed no relevant publication bias (Figure 6). The corresponding funnel plot for subgroup analyses region wise and TNBC cases are depicted in Figure 7 and Figure 8 subsequently.

The sensitivity analysis was performed with Doval, et al. [13] which was described as the low prevalence rate of the TNBC (10.8\%). The overall effect was observed after omitting the data from the primary studies and the combined prevalence obtained was $25.99 \%(95 \% \mathrm{Cl}$, $24.48 \%$ to $27.50 \%$ ).

\section{Discussion}

The Triple negative variant accounts for $12 \%-17 \%$ of the breast cancer subtype in the Western world [27]. It has been found to be more chemo responsive than the different variant of breast cancer $[28,29]$. It also accounts for $70 \%$ of the breast cancer having inherited BRCA1 mutation. The prevalence of aggressive nature of breast cancer in Indian females with poor outcomes was the reason to evaluate the prevalence of TNBC which could to higher in Indian population as a cause for aggressive tumor biology.

Systematic review is the process of integrating empirical evidence from multiple studies to obtain reliable findings and unified conclusions that can answer a specific research question. A systematic review collates studies with design, methods and analysis of the results related to a specific topic [30]. The meta-analysis, a component of systematic review performs quantitative synthesis employing statistical methods. The main goal of meta-analysis is to determine the summary

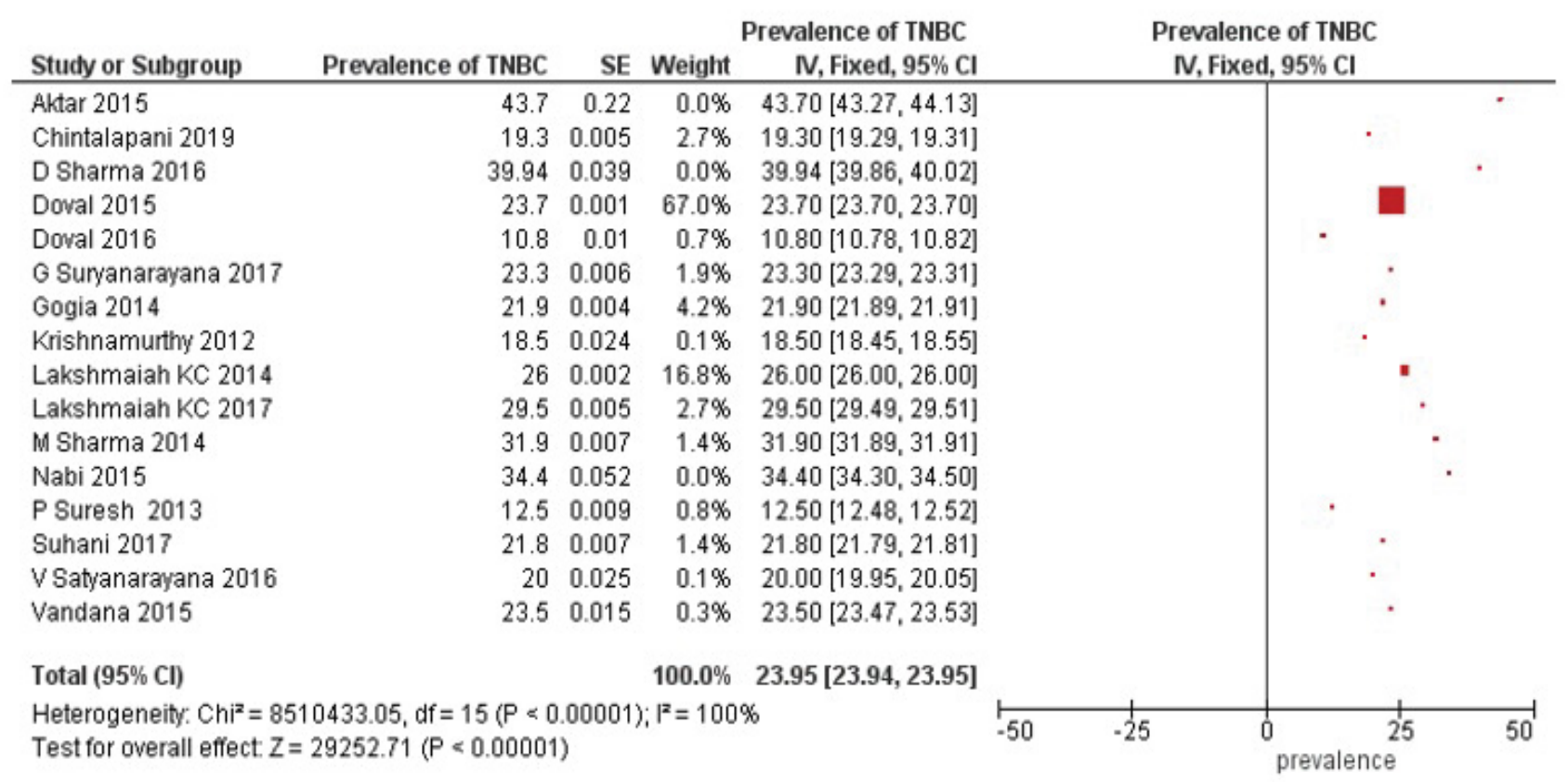

Figure 3: Forest plot illustrating the pooled prevalence rate of TNBC in Indian cohorts with fixed effects model. 


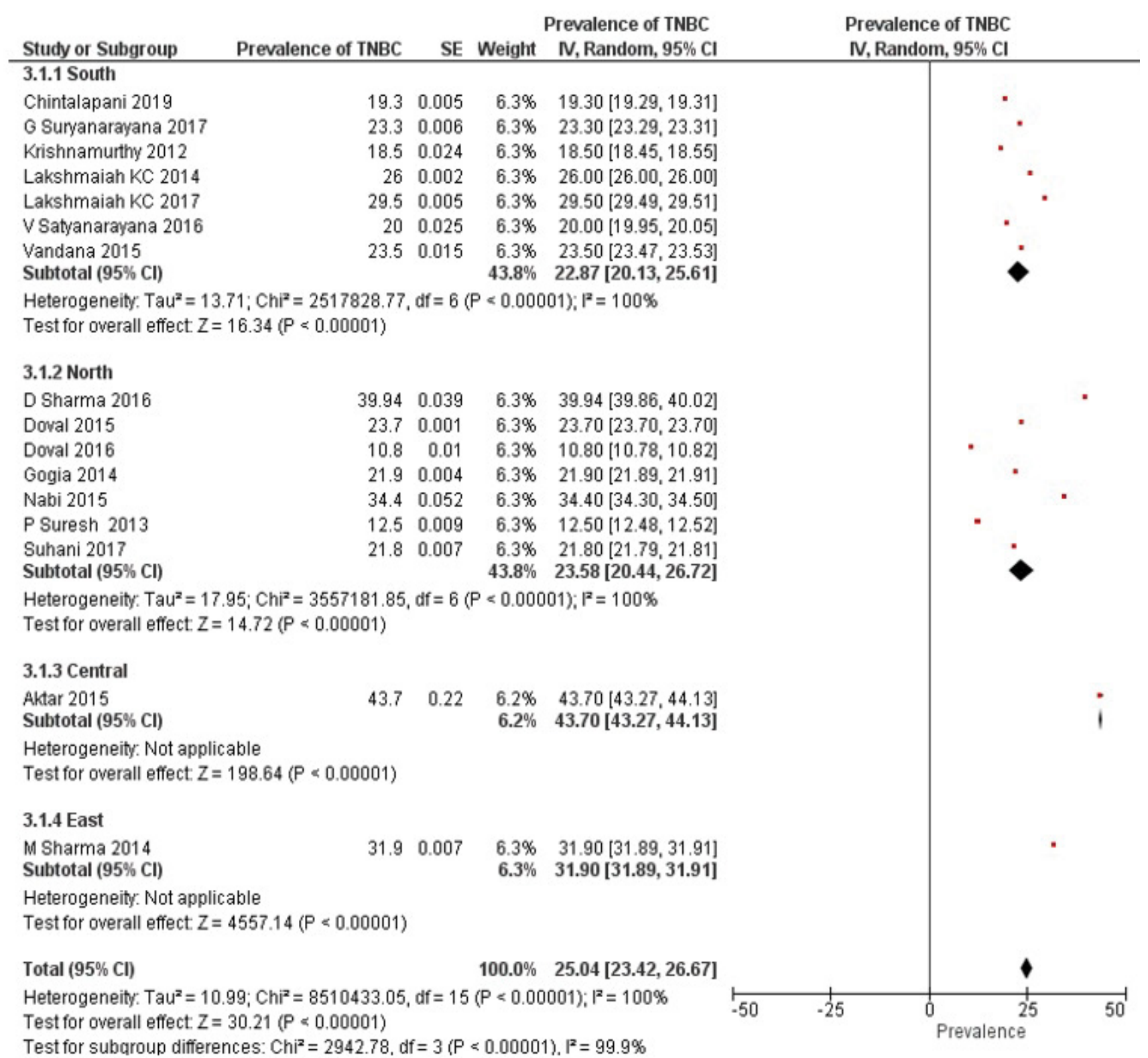

Figure 4: Forest plot-sub group analysis of TNBC prevalence region wise.

estimate of an effect, explore the study heterogeneity and to minimize the publication bias. The meta-analysis literatures with respect to the breast cancer prevalence in Indian scenario are scanty. Availability of the limited studies might be due to scanty in hospital centric records of the patients, digitalized cancer registries and the registration procedure that are interconnected nation-wide.

Systematic review highlighting the various factors interrelated to epidemiology with the breast cancer among the Indian women was carried out by many authors. Malvia, et al. [31] carried out a review by collating the information from several Indian cancer registries and to provide a wider view of epidemiology of breast cancer in the population of India. Madhav, et al. [32] estimated the prevalence, standardized incidence and age-wise mortality rate among Indian women treated with the breast cancer. The study also investigated the effect of diverse demographics, clinic epidemiological and clinicopathological features by subtype analyses. Jayaraj, et al. [33] defined the role of different miRNAs that are associated with the chemo sensitivity or resistance in breast cancer treatment strategies. Pathak, et al. [34] compared the three treatment regimens in neoadjuvant setting and the effectiveness was assessed with different meta-analysis. Chowdhury, et al. [35] summarised the literature on breast feeding and explored its effects with long term and short-term duration of the health outcomes.

Many TNBC related reviews from India has been reported in the literature. The systematic review that underlines the overall prevalence rate of TNBC had been studied by Sandhu, et al. [36] and Kulkarni, et al. [37]. Chatterjee, et al. [38] pointed out some of the issues that had been overlooked in Sandhu, et al. [36]. Thakur, et al. [39] performed a data survey that revealed the 
Prevalence of TNBC

Study or Subgroup Prevalence of TNBC SE Weight IV, Random, $95 \% \mathrm{Cl}$ IV, Random, $95 \% \mathrm{Cl}$

\subsubsection{TNBC cases $<150$}

Aktar 2015

D Sharma 2016

Doval 2016

G Suryanarayana 2017

Krishnamurthy 2012

Lakshmaiah KC 2014

Nabi 2015

Suhani 2017

V Satyanarayana 2016

Vandana 2015

Subtotal $(95 \% \mathrm{Cl})$

$\begin{array}{rrrr}43.7 & 0.22 & 6.2 \% & 43.70[43.27,44.13] \\ 39.94 & 0.039 & 6.3 \% & 39.94[39.86,40.02] \\ 10.8 & 0.01 & 6.3 \% & 10.80[10.78,10.82] \\ 23.3 & 0.006 & 6.3 \% & 23.30[23.29,23.31] \\ 18.5 & 0.024 & 6.3 \% & 18.50[18.45,18.55] \\ 26 & 0.002 & 6.3 \% & 26.00[26.00,26.00] \\ 34.4 & 0.052 & 6.3 \% & 34.40[34.30,34.50] \\ 21.8 & 0.007 & 6.3 \% & 21.80[21.79,21.81] \\ 20 & 0.025 & 6.3 \% & 20.00[19.95,20.05] \\ 23.5 & 0.015 & 6.3 \% & 23.50[23.47,23.53] \\ & & 62.5 \% & 26.19[23.11,29.27]\end{array}$

Heterogeneity: Tau $^{2}=24.76 ; \mathrm{Chi}^{2}=2864768.78, \mathrm{df}=9(\mathrm{P}<0.00001) ; \mathrm{I}^{2}=100 \%$

Test for overall effect: $Z=16.64(P \propto 0.00001)$

\subsubsection{TNBC cases $>150$}

\section{Chintalapani 2019}

Doval 2015

Gogia 2014

Lakshmaiah KC 2017

M Sharma 2014

P Suresh 2013

Subtotal $(95 \% \mathrm{Cl})$

$\begin{array}{rrrr}19.3 & 0.005 & 6.3 \% & 19.30[19.29,19.31] \\ 23.7 & 0.001 & 6.3 \% & 23.70[23.70,23.70] \\ 21.9 & 0.004 & 6.3 \% & 21.90[21.89,21.91] \\ 29.5 & 0.005 & 6.3 \% & 29.50[29.49,29.51] \\ 31.9 & 0.007 & 6.3 \% & 31.90[31.89,31.91] \\ 12.5 & 0.009 & 6.3 \% & 12.50[12.48,12.52] \\ & & 37.5 \% & 23.13[19.88,26.38]\end{array}$

Heterogeneity: Tau $^{2}=16.49 ; \mathrm{Chi}^{2}=5242746.92, \mathrm{df}=5(\mathrm{P}<0.00001) ; \mathrm{I}^{2}=100 \%$

Test for overall effect: $Z=13.95(P \propto 0.00001)$

\section{Total $(95 \% \mathrm{Cl})$}

$100.0 \% 25.04[23.42,26.67]$

Heterogeneity: Tau $^{2}=10.99 ; \mathrm{Chi}^{2}=8510433.05, \mathrm{df}=15(\mathrm{P}<0.00001) ; \mathrm{I}^{2}=100 \%$

Test for overall effect: $Z=30.21$ ( $P<0.00001$ )

Test for subqroup differences: $\mathrm{Ch}^{2}=1.79, \mathrm{df}=1(\mathrm{P}=0.18), \mathrm{I}^{2}=44.1 \%$

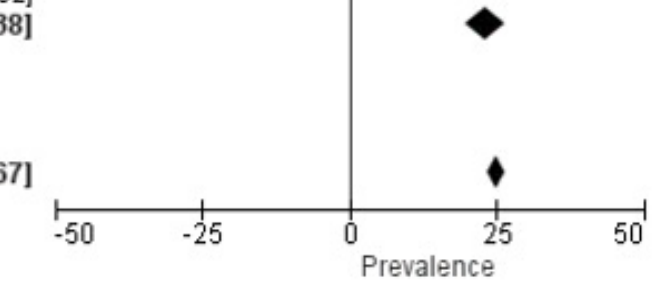

Figure 5: TNBC cases are subgroup into two categories: Cases $<150$ and cases $>150$. Study heterogeneity and subgroup differences is presented using forest plot.

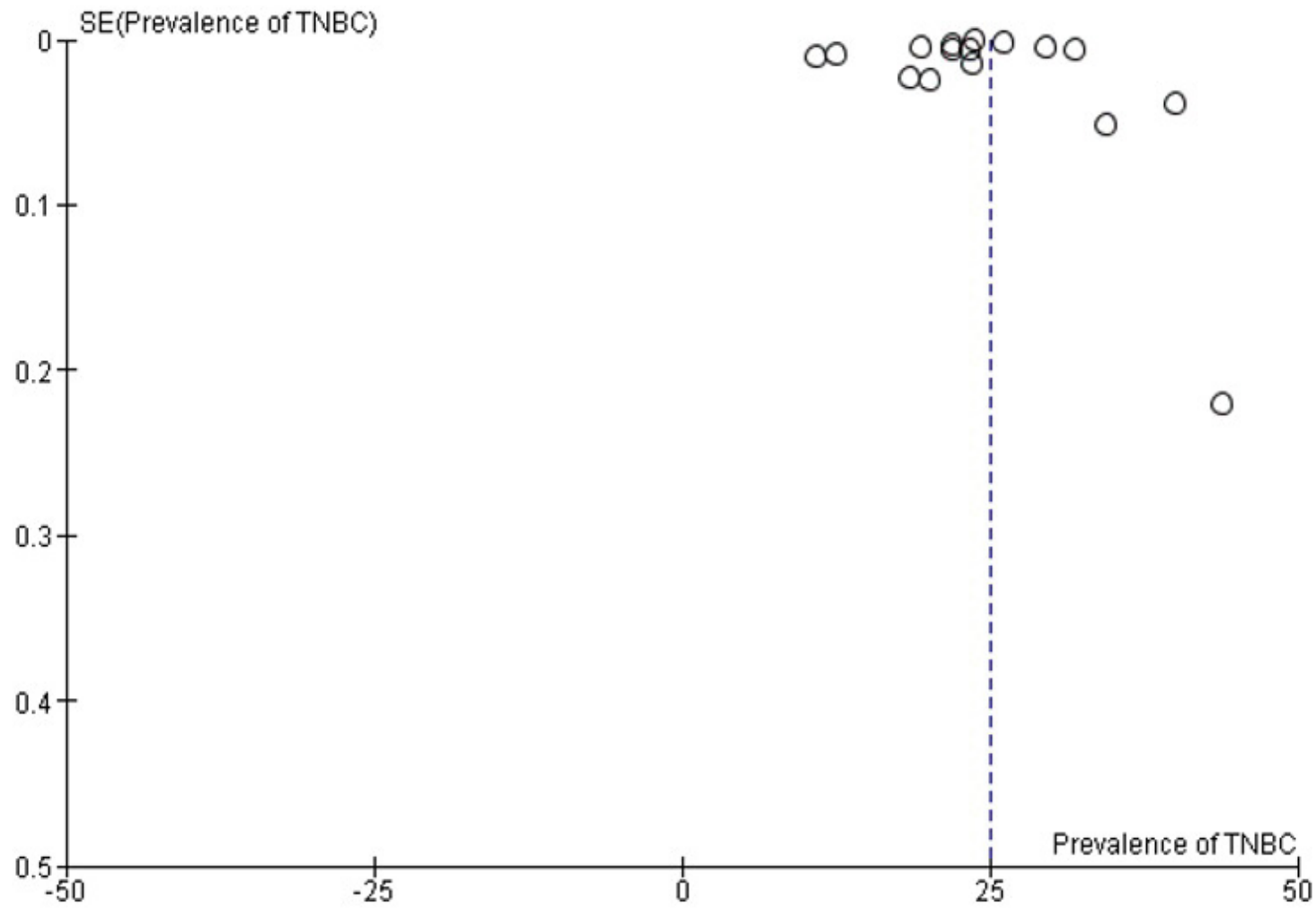

Figure 6: Funnel plot illustrating the study specific TNBC prevalence in X-axis and standard error in Y-axis. 


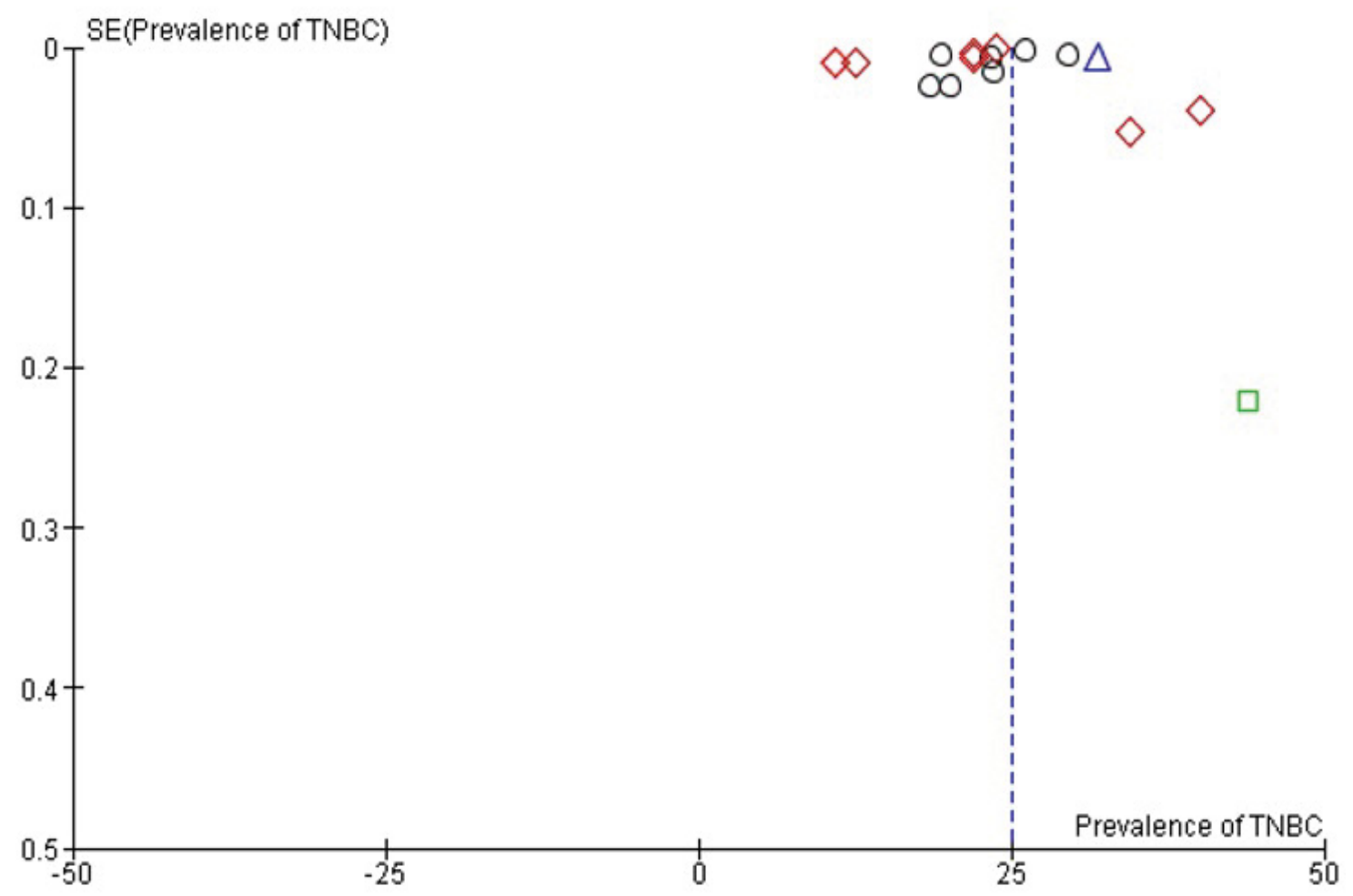

Subgroups

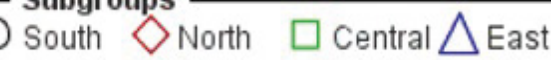

Figure 7: Funnel plot - TNBC prevalence rate region wise with corresponding standard error.

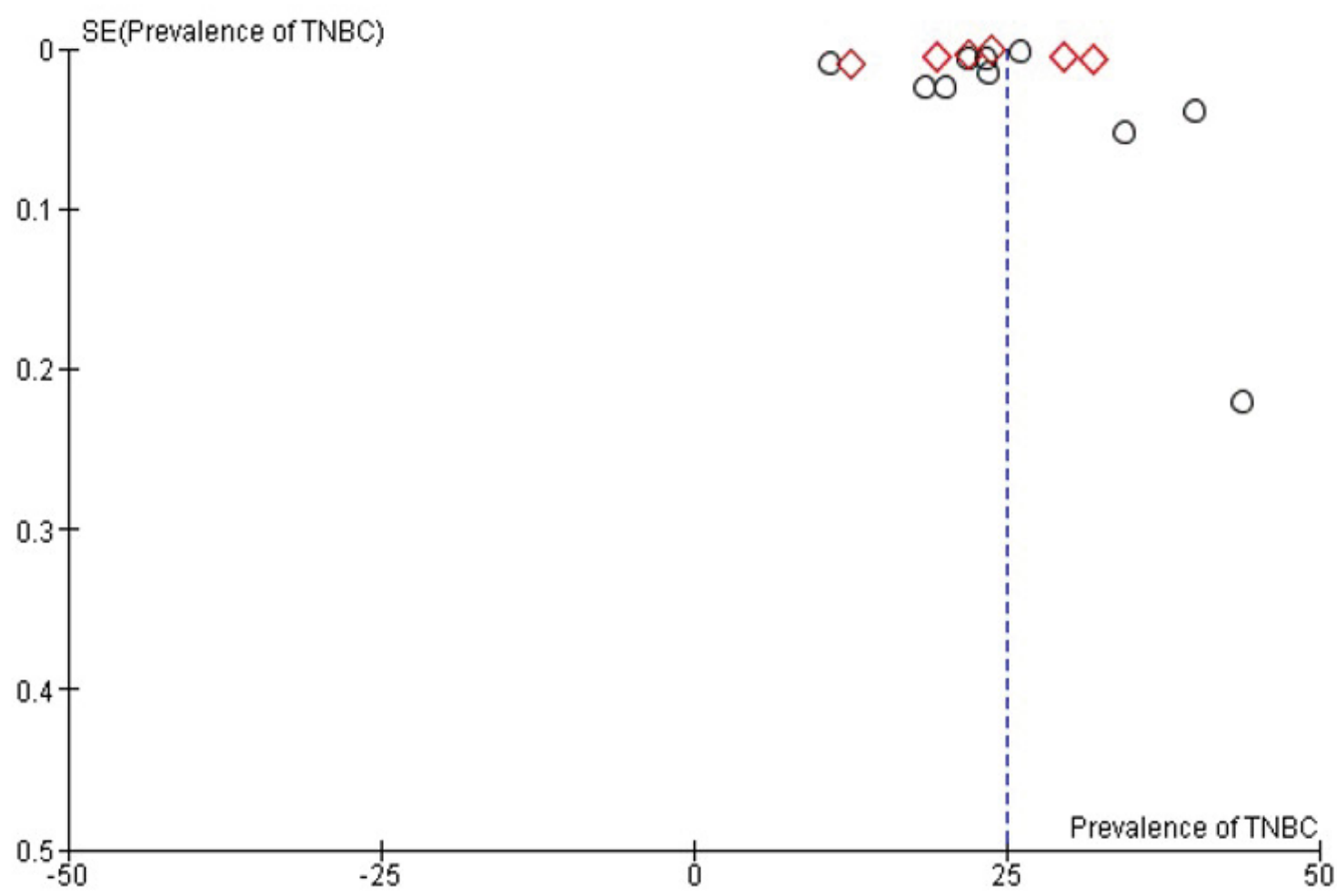

Subgroups

TNBC cases $<150 \diamond$ TNBC cases $>150$

Figure 8: Funnel plot - prevalence of TNBC cases into two groups: Case $<150$ and cases $>150$ in X-axis and standard error in $\mathrm{Y}$-axis. 
higher proportion of the TNBC in India which is quite consistent with present study. There is a considerable increase in prevalence of the TNBC patients ranging from $10 \%$ to $43 \%$ which is evident from the various literatures reported over a decade [10-25]. These studies also support our result of $25.04 \%$ prevalence of the TNBC which is greater in India when compared to western literature [40]. The prevalence rate of these studies had regional differences too, hence there was a need for getting studies from all regions of India in systematic review and meta-analysis to take care of these variation while calculating the pooled Pan India prevalence. High value of Chi Square in regional sub group analysis was also an indicator of gross heterogeneity in data processed. As majority of studies included were from south and north India, so the generalisability of present prevalence rate is questionable.

The present study selected only PubMed and Google scholar studies from India while in reality, lot of studies are published in Indian Journals which are not PubMed or Google scholar indexed hence there could be a publication bias but there is authenticity of data and strong methodology in these indexed journals which makes our study more scientifically based and results more valid. The duration of 10 years for selection of study is justified with the reason that changing tumour biology and scarcity of Immunohistochemistry facilities in first decade of this century in Indian scenario.

\section{Conclusion}

TNBC is an aggressive variant of breast cancer appears to affect younger patients with poor prognosis and one of the reasons for such aggressive disease could be due to higher prevalence of $25.04 \%$ as concluded from the present meta-analysis. Based on our results, it is necessary to carry out meta-analysis from all regions of vast geography of India and if feasible calculating regional prevalence to identify population at greater risk. Considering higher prevalence of TNBC from existing data there is a need to carry out more research in treatment modalities of TNBC to improve overall short and long-term clinical outcomes.

\section{Financial Support}

Nil.

\section{Conflicts of Interest}

The authors declare that there are no conflicts of interest in this paper.

\section{Sources of Support}

Nil.

\section{Author Contributions}

Suvobrata Sarkar: Conceptualization, data curation, investigation, software, writing original draft; Dr. Murtaza Akhtar: Formal analysis, data curation, investigation, editing the original draft.

\section{References}

1. Johnson RH, Anders CK, Litton JK, Ruddy KJ, Bleyer A (2018) Breast cancer in adolescents and young adults. Pediatr Blood Cancer 65: e27397.

2. Ferlay J, Soerjomataram I, Ervik M (2013) GLOBOCAN 2012 v1.0, cancer incidence and mortality worldwide: IARC Cancer Base No. 11 [Internet]. International Agency for Research on Cancer, Lyon, France.

3. Bray F, Ren JS, Masuyer E, Ferlay J (2013) Global estimates of cancer prevalence for 27 sites in the adult population in 2008. Int J Cancer 132: 1133-1145.

4. Chaurasia V, Pal S (2014) A novel approach for breast cancer detection using data mining techniques. International Journal of Innovative Research in Computer and Communication Engineering 2: 2456-2465.

5. Hutton B, Salanti G, Caldwell DM, Chaimani A, Schmid CH, et al. (2015) The PRISMA extension statement for reporting of systematic reviews incorporating network meta-analyses of health care interventions: Checklist and explanations. Ann Intern Med 162: 777-784.

6. Harris JD, Quatman CE, Manring MM, Siston RA, Flanigan DC (2014) How to write a systematic review. Am J Sports Med 42: 2761-2768.

7. Liberati A, Altman DG, Tetzlaff J, Mulrow C, Gøtzsche PC, et al. (2009) The PRISMA statement for reporting systematic reviews and meta-analyses of studies that evaluate health care interventions: Explanation and elaboration. J Clin Epidemiol 62: e1-e34.

8. Jackson D, White IR, Riley RD (2012) Quantifying the impact of between-study heterogeneity in multivariate meta-analyses. Stat Med 31: 3805-3820.

9. Review Manager (RevMan) (2014) Computer program: Version 5.3. The Nordic Cochrane Centre, The Cochrane Collaboration, Copenhagen.

10. Suhani, Parshad R, Kazi M, Seenu V, Mathur S, et al. (2017) Triple-negative breast cancers: Are they always different from nontriple-negative breast cancers? An experience from a tertiary center in India. Indian J Cancer 54: 658-663.

11. Lakshmaiah KC, Anand A, Babu KG, Dasappa L, Jacob LA, et al. (2017) Role of taxanes in triple-negative breast cancer: A study from tertiary cancer center in South India. World J Oncol 8: 110-116.

12. Sharma D, Singh G (2016) An institutional analysis of clinicopathological features of triple negative breast cancer. Indian J Cancer 53: 566-568.

13. Chandra D, Suresh $P$, Sinha R, Azam S, Batra U, et al. (2016) Eight year survival analysis of patients with triple negative breast cancer in India. Asian Pac J Cancer Prev 17: 2995-2999.

14. Akhtar M, Dasgupta S, Rangwala M (2015) Triple negative breast cancer: An Indian perspective. Breast Cancer (Dove Med Press) 7: 239-243.

15. Doval DC, Sharma A, Sinha R, Kumar K, Dewan AK, et al. (2015) Immunohistochemical profile of breast cancer patients at a tertiary care hospital in New Delhi, India. Asian Pac J Cancer Prev 16: 4959-4964.

16. Nabi MG, Ahangar A, Wahid MA, Kuchay S (2015) Clinicopathological comparison of triple negative breast cancers with non-triple negative breast cancers in a hospital in North India. Niger J Clin Pract 18: 381-386.

17. Lakshmaiah KC, Das U, Suresh TM, Lokanatha D, Babu 
GK, et al. (2014) A study of triple negative breast cancer at a tertiary cancer care center in Southern India. Ann Med Health Sci Res 4: 933-937.

18. Gogia A, Raina V, Deo SVS, Shukla NK, Mohanti BK (2014) Triple-negative breast cancer: An institutional analysis. Indian J Cancer 51: 163-166.

19. Sharma M, Sharma JD, Sarma A, Ahmed S, Kataki AC, et al. (2014) Triple negative breast cancer in people of North East India: Critical insights gained at a regional cancer centre. Asian Pac J Cancer Prev 15: 4507-4511.

20. Suresh P, Batra U, Doval DC (2013) Epidemiological and clinical profile of triple negative breast cancer at a cancer hospital in North India. Indian J Med Paediatr Oncol 34: 89-95.

21. Krishnamurthy $S$, Poornima R, Challa VR, Goud YGB (2012) Triple negative breast cancer - our experience and review. Indian J Surg Oncol 3: 12-16.

22. Chintalapani SR, Bala S, Konatam ML, Gundeti S, Kuruva SP, et al. (2019) Triple-negative breast cancer: Pattern of recurrence and survival outcomes. Indian $\mathrm{J}$ Med Paediatr Oncol 40: 67-72.

23. Raju GS, Shantappa R, Jena S (2017) Triple negative breast cancer: A therapeutic challenge. International Journal of Medical and Health Research 3: 12-15.

24. Satyanarayan V, Ashok A (2016) Triple negative breast cancer- experience at tertiary care centre, South India. International Journal of Current Research 8: 42382-42383.

25. Gaopande VL, Joshi SS, Kulkarni MM, Dwivedi SS (2015) A clinicopathologic study of triple negative breast cancer. J Sci Soc 42: 12-15.

26. User manual in STATA for meta-analysis.

27. Foulkes WD, Smith IE, Reis-Filho JS (2010) Triple-negative breast cancer. N Engl J Med 363: 1938-1948.

28. Carey LA, Dees EC, Sawyer L, Gatti L, Moore DT, et al. (2007) The triple negative paradox: Primary tumor chemosensitivity of breast cancer subtypes. Clin Cancer Res 13: 2329-2334.

29. Liedtke C, Mazouni C, Hess KR, André F, Tordai A, et al. (2008) Response to neoadjuvant therapy and long-term survival in patients with triple-negative breast cancer. $\mathrm{J}$ Clin Oncol 26: 1275-1281.

30. Kang H (2015) Statistical considerations in meta-analysis. Hanyang Med Rev 35: 23-32.
31. Malvia S, Bagadi SA, Dubey US, Saxena S (2017) Epidemiology of breast cancer in Indian women. Asia Pac J Clin Oncol 13: 289-295.

32. Madhav MR, Nayagam SG, Biyani K, Pandey V, Kamal DG, et al. (2018) Epidemiologic analysis of breast cancer incidence, prevalence, and mortality in India: Protocol for a systematic review and meta-analyses. Medicine (Baltimore) 97: e13680.

33. Jayaraj R, Nayagam SG, Kar A, Sathyakumar S, Mohammed $\mathrm{H}$, et al. (2019) Clinical theragnostic relationship between drug-resistance specific miRNA expressions, chemotherapeutic resistance, and sensitivity in breast cancer: A systematic review and meta-analysis. Cells 8: 1250.

34. Pathak M, Dwivedi SN, Deo SVS, Thakur B, Sreenivas V (2018) Neoadjuvant chemotherapy regimens in treatment of breast cancer: A systematic review and network metaanalysis protocol. Syst Rev 7: 89.

35. Chowdhury R, Sinha B, Sankar MJ, Taneja S, Bhandari N, et al. (2015) Breastfeeding and maternal health outcomes: A systematic review and meta-analysis. Acta Paediatr 104: 96-113.

36. Sandhu GS, Erqou S, Patterson H, Mathew A (2016) Prevalence of triple-negative breast cancer in India: Systematic review and meta-analysis. J Glob Oncol 2: 412421.

37. Kulkarni A, Kelkar DA, Parikh N, Shashidhara LS, Koppiker CB, et al. (2020) Meta-analysis of prevalence of triplenegative breast cancer and its clinical features at incidence in Indian patients with breast cancer. JCO Glob Oncol 6: 1052-1062.

38. Chatterjee S, Arun I, Agrawal S, Arunsingh M, Indranil M, Ahmed R (2016) Immunohistochemistry heterogeneity in reported breast cancer demographics from India: Triplenegative breast cancer rates could be lower than suggested in pooled meta-analysis. J Glob Oncol 3: 180-181.

39. Thakur KK, Bordoloi D, Kunnumakkara AB (2018) Alarming burden of triple-negative breast cancer in India. Clin Breast Cancer 18: e393-e399.

40. Dent R, Trudeau M, Pritchard KI, Hanna WM, Kahn HK, et al. (2007) Triple-negative breast cancer: Clinical features and patterns of recurrence. Clin Cancer Res 13: 4429-4434. 